\title{
Simulations of neutrino and muon interaction in matter for geological structures radiography
}

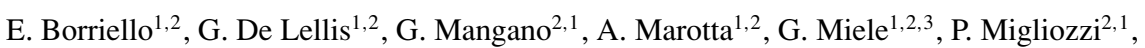

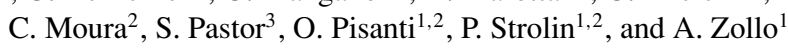 \\ ${ }^{1}$ Università "Federico II", Dipartimento di Scienze Fisiche, Complesso Universitario di Monte S.Angelo, \\ Via Cithia, 80126, Napoli, Italy \\ ${ }^{2}$ INFN-Sezione di Napoli-Complesso Universitario di Monte S.Angelo, Via Cithia, 80126, Napoli, Italy \\ ${ }^{3}$ Instituto de Física Corpuscular (CSIC-Universitat de València), Ed. Institutos de Investigación, \\ Apartado de Correos 22085, E-46071 València, Spain
}

(Received December 1, 2008; Revised June 16, 2009; Accepted June 17, 2009; Online published February 22, 2010)

\begin{abstract}
Neutrino and muon radiography seems to provide a method complementary to the more conventional seismic studies for getting information on the very deep geological structures. Here we describe the status of the simulations of neutrino and muon interaction in matter.
\end{abstract}

Key words: High energy cosmic rays, neutrinos, Earth radial density, muons.

\section{Introduction}

The internal density profile and shape of Earth is commonly studied by indirect physical methods, starting from the 1793 deduction by Cavendish that the Earth must have a dense core, based on a gravitational calculation, to the current methods which use sismic wave propagation, studies of the vibrational modes of the Earth as an elastic body, or temperature constraints. However, these studies are still subject to intrinsic ambiguities (Aki and Richards, 1980; Lay and Wallace, 1995). For these reasons, independent measurements of the density profile would be of considerable value and in the last years complementary methods for Earth radiography were pursued, by using cosmic beams of neutrinos and muons. The principle of tomography by energetic beams is essentially the same as X-ray tomography, except for substituting penetrating particles in place of $\mathrm{X}$ rays. By measuring their absorption along different paths through a solid body, one can deduce the nucleon density in the interior of the object.

The Earth's tomography with ultra-high energy cosmic neutrinos seems to provide a viable independent determination of the Earth's internal structure (Jain et al., 1999; Reynoso and Sampayo, 2004), giving some information on its internal density distribution. On the other side, cosmic ray muon radiography can be applied to km-size objects located at elevations above where the detector is placed (Ambrosio et al., 1995; Nagamine et al., 1995; Tanaka et al., 2003, 2005, 2007a-c, 2008; Tanaka and Yokoyama, 2008).

The idea of neutrino tomography is based on the fact that Earth becomes opaque to neutrinos of energy exceeding $\sim 10 \mathrm{TeV}$, since at this energy the diameter of Earth corre-

Copyright (c) The Society of Geomagnetism and Earth, Planetary and Space Sciences (SGEPSS); The Seismological Society of Japan; The Volcanological Society of Japan; The Geodetic Society of Japan; The Japanese Society for Planetary Sciences; TERRAPUB.

doi: $10.5047 /$ eps.2009.06.006 sponds to about one absorption length. Such neutrinos are produced by collisions of cosmic rays with the Earth atmosphere, and interact during their travel in such a way that the charged leptons produced, essentially muons, could emerge from the surface and be detected by a $\mathrm{km}^{3}$ Neutrino Telescope (NT). In this concern, after the first generation of telescopes which has proved the feasibility of the Cerenkov detection technics under deep water (Balkanov et al., 1999) and ice (Ahrens et al., 2002) by detecting atmospheric neutrinos, we are likely approaching the first detections of astrophysical neutrinos at the IceCube (Ahrens et al., 2004) telescope, being completed at the South Pole, and possibly at the smaller ANTARES (Spurio, 2006) telescope under construction in the Mediterranean. Moreover, ANTARES as well as NESTOR (Aggouras et al., 2006) and NEMO (Migneco et al., 2008) are involved in R\&D projects aimed at the construction of a $\mathrm{km}^{3} \mathrm{NT}$ in the deep water of the Mediterranean sea, coordinated in the European network KM3NeT (Katz, 2006).

On the other side, cosmic ray muons are also generated from cosmic rays in the atmosphere and arrive at angles ranging from vertical to horizontal (Thompson and Whalley, 1975) with a smaller number of neutrino-induced muons directed upward. These particles are highly penetrating and a typical horizontally-arriving cosmic-ray muon with an energy of $1 \mathrm{TeV}$ penetrates $2.6 \mathrm{~km}$ of water. Thus, cosmic ray muon radiography can be applied to Earth structures with size of the order of a $\mathrm{km}$, placing a detector with a smaller area than a NT $\left(\sim \mathrm{m}^{2}\right)$ near a volume that is higher in elevation adjacent to the detector.

In both of these technics, full Monte Carlo simulations are needed for the extraction of physical parameters from the detected numbers of events. In the following we will report on the status of studies, aiming to simulate the interaction of neutrinos inside the Earth and the propagation of their parents leptons inside matter. 


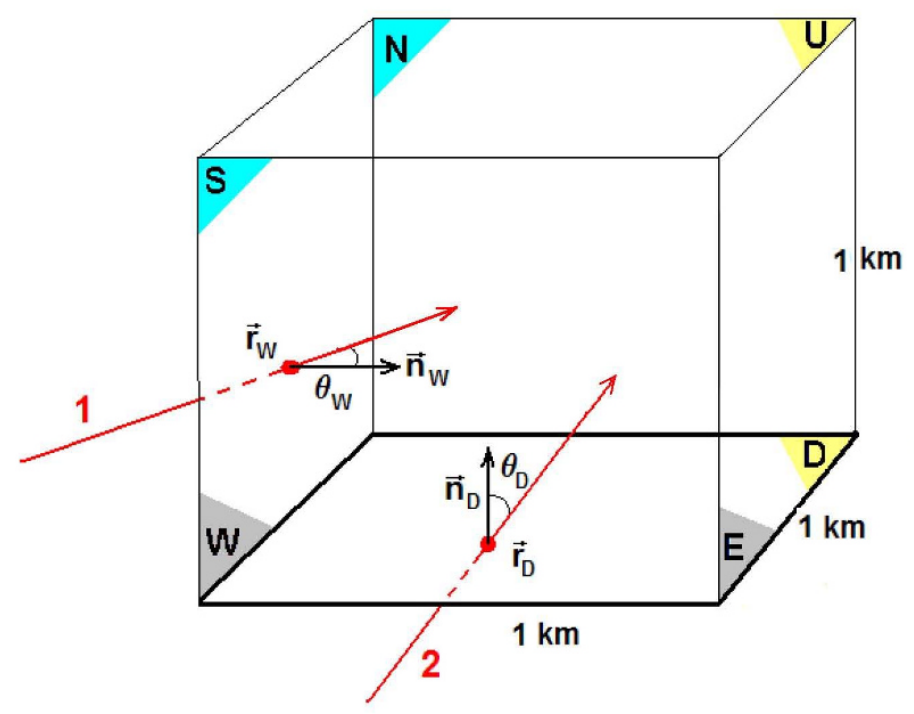

Fig. 1. The angle definition and the fiducial volume of a $\mathrm{km}^{3} \mathrm{NT}$.

\section{Earth's Tomography with Neutrinos and Muons}

In order to understand how the number of charged lepton events at a $\mathrm{km}^{3} \mathrm{NT}$ depends on the density of matter crossed by $\mathrm{HE}$ neutrinos, let us remind the formalism developed in Cuoco et al. (2007). In the following we will refer our calculation to $\mathrm{km}^{3} \mathrm{NT}$ placed at NEMO site.

We define the $\mathrm{km}^{3}$ NT fiducial volume as that bounded by the six lateral surfaces $\Sigma_{a}$ (the subindex $a=\mathrm{D}, \mathrm{U}, \mathrm{S}$, $\mathrm{N}, \mathrm{W}$, and E labels each surface through its orientation: Down, Up, South, North, West, and East), and indicate with $\Omega_{a} \equiv\left(\theta_{a}, \phi_{a}\right)$ the generic direction of a track entering the surface $\Sigma_{a}$. The scheme of the NT fiducial volume and two examples of incoming tracks are shown in Fig. 1.

Let $\mathrm{d} \Phi_{\nu} /\left(\mathrm{d} E_{\nu} \mathrm{d} \Omega_{a}\right)$ be the differential flux of UHE $v_{\mu}+$ $\bar{v}_{\mu}$. The number per unit time of $\mu$ leptons emerging from the Earth surface and entering the NT with energy $E_{\tau}$ is given by

$$
\begin{aligned}
\frac{\mathrm{d} N_{\mu}}{\mathrm{d} t}= & \sum_{a} \int \mathrm{d} \Omega_{a} \int \mathrm{d} S_{a} \int \mathrm{d} E_{\nu} \frac{\mathrm{d} \Phi_{\nu}\left(E_{\nu}, \Omega_{a}\right)}{\mathrm{d} E_{\nu} \mathrm{d} \Omega_{a}} \\
& \times \int \mathrm{d} E_{\mu} \cos \left(\theta_{a}\right) k_{a}^{\mu}\left(E_{\nu}, E_{\mu} ; \vec{r}_{a}, \Omega_{a}\right) .
\end{aligned}
$$

The kernel $k_{a}^{\mu}\left(E_{v}, E_{\mu} ; \vec{r}_{a}, \Omega_{a}\right)$ is the probability that an incoming $v_{\mu}$ crossing the Earth, with energy $E_{v}$ and direction $\Omega_{a}$, produces a $\mu$-lepton which enters the NT fiducial volume through the lateral surface $\mathrm{d} S_{a}$ at the position $\vec{r}_{a}$ with energy $E_{\mu}$ (see Fig. 1 for the angle definition).

As already shown in Cuoco et al. (2007) (the interested reader can find in Miele et al. (2006) more details on the equations reported in the following), a typical event corresponds to the simultaneous fulfillment of the following conditions:

1) A $v_{\mu}$ with energy $E_{v}$ travels over a distance $z$ through the Earth before interacting. The corresponding prob- ability $P_{1}$ is given by

$$
P_{1}=\exp \left\{-\frac{z}{\lambda_{\mathrm{CC}}^{v}\left(E_{v}\right)}\right\}
$$

with

$$
\lambda_{\mathrm{CC}}^{\nu}\left(E_{v}\right)=\frac{1}{\sigma_{\mathrm{CC}}^{\nu N}\left(E_{v}\right) \varrho_{\mathrm{r}} N_{\mathrm{A}}},
$$

where $N_{\mathrm{A}}$ is the Avogadro number and $\varrho_{\mathrm{r}}$ is the Earth density assumed to be constant.

2) The neutrino produces a $\mu$ in the interval $z, z+\mathrm{d} z$, the probability of such an event being

$$
\mathrm{d} P_{2}=\frac{\mathrm{d} z}{\lambda_{\mathrm{CC}}^{\nu}\left(E_{v}\right)} \text {. }
$$

3) The produced $\mu$ emerges from the Earth rock with an energy $E_{\mu}^{\prime}$. This happens with a probability

$$
\begin{aligned}
P_{3}= & \exp \left\{-\frac{m_{\mu}}{c \tau_{\mu} \beta_{\mu} \varrho_{\mathrm{r}}}\left(\frac{1}{E_{\mu}^{\prime}}-\frac{1}{E_{\mu}^{0}\left(E_{\nu}\right)}\right)\right\} \\
& \times \delta\left(E_{\mu}^{\prime}-E_{\mu}^{0}\left(E_{\nu}\right) e^{-\beta_{\mu} \varrho_{\mathrm{r}}\left(z_{\mathrm{r}}-z\right)}\right) .
\end{aligned}
$$

4) Finally, the $\mu$ lepton emerging from the Earth rock propagates in water and enters the NT fiducial volume through the lateral surface $\Sigma_{a}$ at the point $\vec{r}_{a}$ with energy $E_{\mu}$. The corresponding survival probability is

$$
\begin{aligned}
P_{4}= & \exp \left\{-\frac{m_{\mu}}{c \tau_{\mu} \beta_{\mu} \varrho_{\mathrm{w}}}\left(\frac{1}{E_{\mu}}-\frac{1}{E_{\mu}^{\prime}}\right)\right\} \\
& \times \delta\left(E_{\mu}-E_{\mu}^{\prime} e^{-\beta_{\mu} \rho_{\mathrm{w}} z_{\mathrm{w}}}\right),
\end{aligned}
$$

where $\varrho_{\mathrm{w}}$ stands for the water density and $z_{\mathrm{w}}\left(\vec{r}_{a}, \Omega_{a}\right)$ represents the total length in water before arriving to the fiducial volume for a given track entering the lateral surface $\Sigma_{a}$ at the point $\vec{r}_{a}$ and with direction $\Omega_{a}$. 

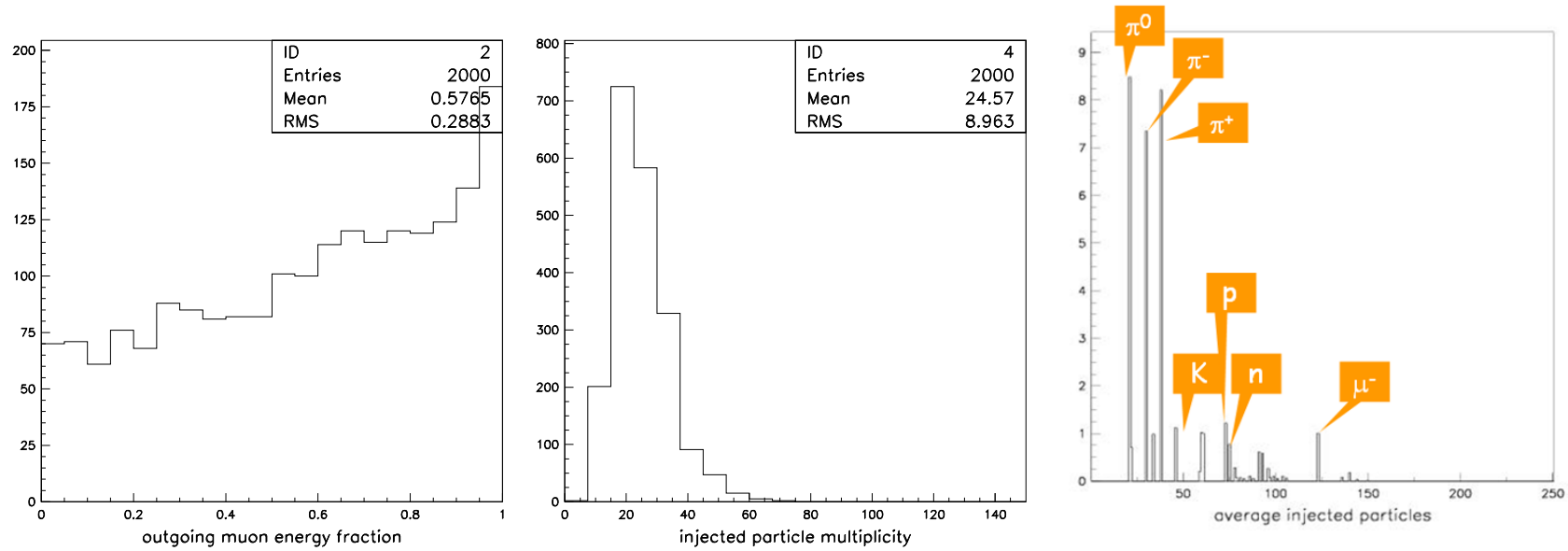

Fig. 2. From left to right, the plots show the outgoing muon energy fraction and the multiplicity distribution of the first interaction particles, respectively, for the Charge Current (CC) interaction of 2000 neutrino events simulated by HERWIG at the energy of $10^{4} \mathrm{GeV}$, and finally the type of particles produced at the energy of $10^{5} \mathrm{GeV}$.

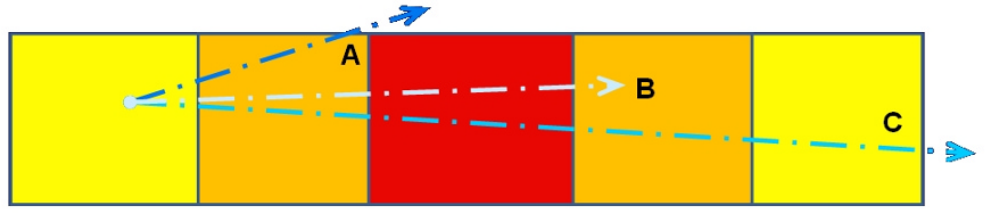

Fig. 3. Type of tracks considered in the simulation with GEANT4.

Collecting together the different probabilities in Eqs. (2), (4), (5) and (6), we have

$$
k_{a}^{\mu}\left(E_{\nu}, E_{\mu} ; \vec{r}_{a}, \Omega_{a}\right)=\int_{0}^{z_{\mathrm{r}}} \mathrm{d} z \int_{0}^{E_{\tau}^{0}\left(E_{v}\right)} \mathrm{d} E_{\tau}^{\prime} P_{1} P_{2} P_{3} P_{4} .
$$

Now, the Earth density profile enters via the matter density, $\varrho_{\mathrm{r}}$, in most of the previous expressions, and thus one must expect a certain sensitivity of the number of detected events to the characteristic of the Earth model.

Similar considerations apply to the case of muon radiography, with the difference that now the primary particles considered are the muons produced through the cosmic ray interaction with the Earth atmosphere. Such muons enter Earth and lose energy by ionization and radiative processes: bremsstrahlung, direct production of $e^{+} e^{-}$pairs, and photonuclear interaction. The total muon energy loss may be expressed as a function of the amount of matter traversed as

$$
-\frac{\mathrm{d} E_{\mu}}{\mathrm{d} x}=\alpha+\beta E_{\mu},
$$

where $\alpha$ is the ionization loss and $\beta$ is the fractional energy loss by the three radiation processes (Gaisser and Stanev, 2008). These parameters are quite sensitive to the chemical composition of the rock.

\section{Simulation of Neutrino and Lepton Propaga- tion Inside Matter}

Our work aims to the development of Monte Carlo methods for simulating neutrino and muon interaction in matter. A possible line towards this purpose is based on the combination of two existing Monte Carlo, the HERWIG hadron generator (Corcella et al., 2001) to simulate neutrino interactions inside the Earth, and GEANT4 (Geometry ANd Tracking) (Agostinelli et al., 2003; Allison et al., 2006, http://www.geant4.org) to simulate the propagation of the produced muons in matter.

HERWIG (Corcella et al., 2001) is an event generator for high-energy processes particularly suited for detailed simulation of QCD parton showers. It provides simulation of hard lepton-lepton, lepton-hadron and hadron-hadron scattering and soft hadron-hadron collisions within a single package. In particular, HERWIG can simulate the neutrino interaction and, in a previous work (Ambrosio et al., 2003), it was already combined with the Monte Carlo CORSIKA (Heck et al., 1998), by some of the present authors, to develop a new version of CORSIKA capable of simulating atmospheric showers induced by neutrinos.

Geant 4 is a toolkit for the simulation of the passage of particles through matter. Its areas of application include high energy, nuclear and accelerator physics, as well as studies in medical and space science.

In the simulations here described, we have generated a large number of tracks crossing the NEMO site by means of a detailed Digital Elevation Map of the under-water Earth surface, which is available from the Global Relief Data survey (ETOPO2): a grid of altimetry measurements with a vertical resolution of $1 \mathrm{~m}$ averaged over cells of 2 minutes of latitude and longitude. The Earth is described by a simplified version of the Preliminary Reference Earth Model (PREM) (Dziewonski, 1971), with three density regions: crust, mantle, and core.

We first describe the characteristic of the HERWIG sim- 

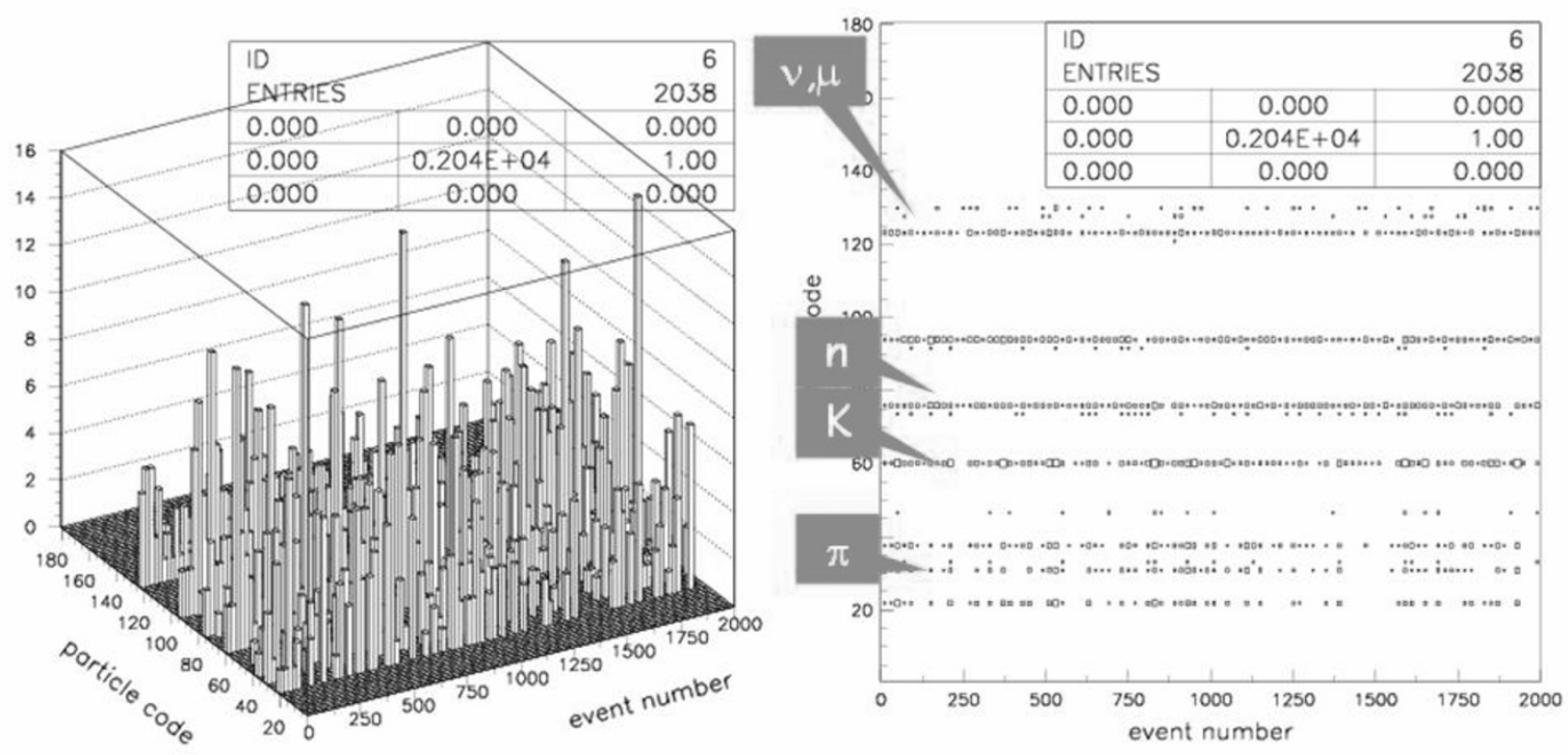

Fig. 4. Number and type of the particles arriving to a NT in the first 2000 events of a GEANT4 run for a primary neutrino of energy $10^{4} \mathrm{GeV}$ (see text).

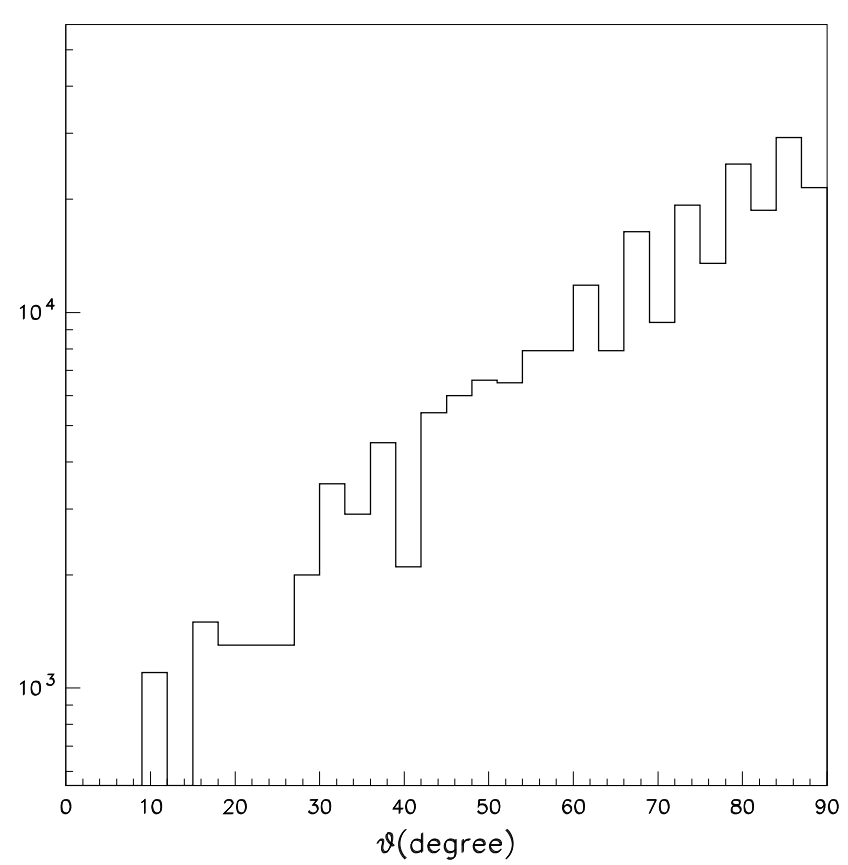

Fig. 5. Zenith angle distribution of neutrino induced events in a NT for a muon energy threshold of $1 \mathrm{TeV}$ and the PREM. Neutrinos coming from below correspond to $\theta=0$.

ulation. In Fig. 2 we show from left to right the outgoing muon energy fraction and the multiplicity distribution of the first interaction particles, respectively, for the Charge Current (CC) interaction of 2000 neutrino events simulated by HERWIG at the energy of $10^{4} \mathrm{GeV}$, while last plot on the right show the type of particles produced.

The first interaction particles produced by HERWIG are extracted and injected in subsequent runs of GEANT4. The GEANT4 simulation is performed following particle tracking in a material with the rock characteristic, divided into

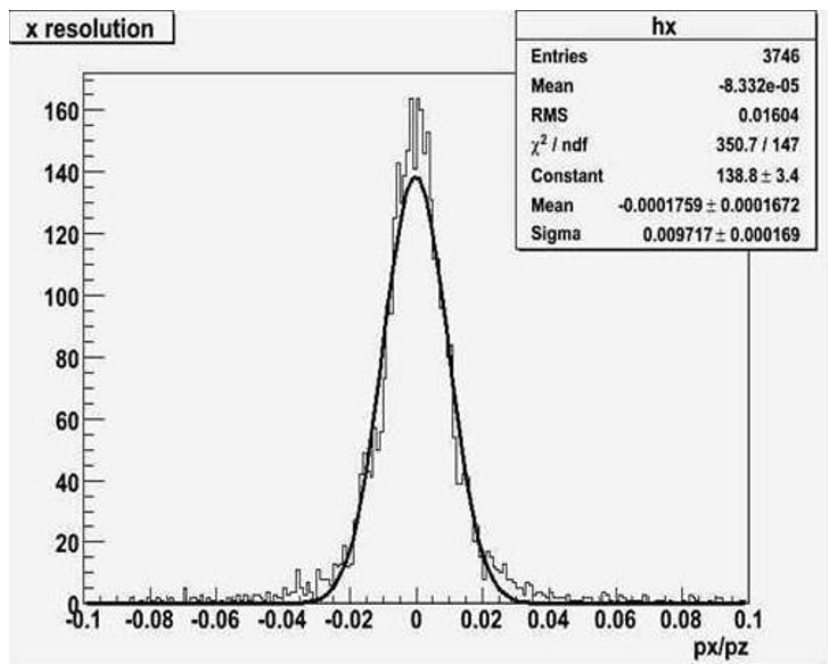

Fig. 6. Angular resolution of the outgoing muons surviving $1 \mathrm{~km}$ of rock with density $2.6 \mathrm{~g} / \mathrm{cm}^{3}$.

a maximum of 5 different zones with 3 possible densities (see Fig. 3). This corresponds to three different kind of neutrino tracks inside the Earth: the ones which go 1) through the core, 2) through mantle and crust, and 3) only through the crust. The tracking stops if the track leaves the tracker (track A in Fig. 3) or if the energy of the particle is less than $1 \mathrm{GeV}$ (track B in Fig. 3) so to make the simulation faster and take into account only interesting particles.

In order to test the results coming from the link of HERWIG and GEANT4, we developed also a simplified Monte Carlo, which simulates neutrino interaction in Earth, and propagates the outgoing muon, taking into account the phenomenon of neutrino regeneration by NC interaction and muon energy loss in matter. The number of neutrinos injected at each angular bin was scaled according to the 


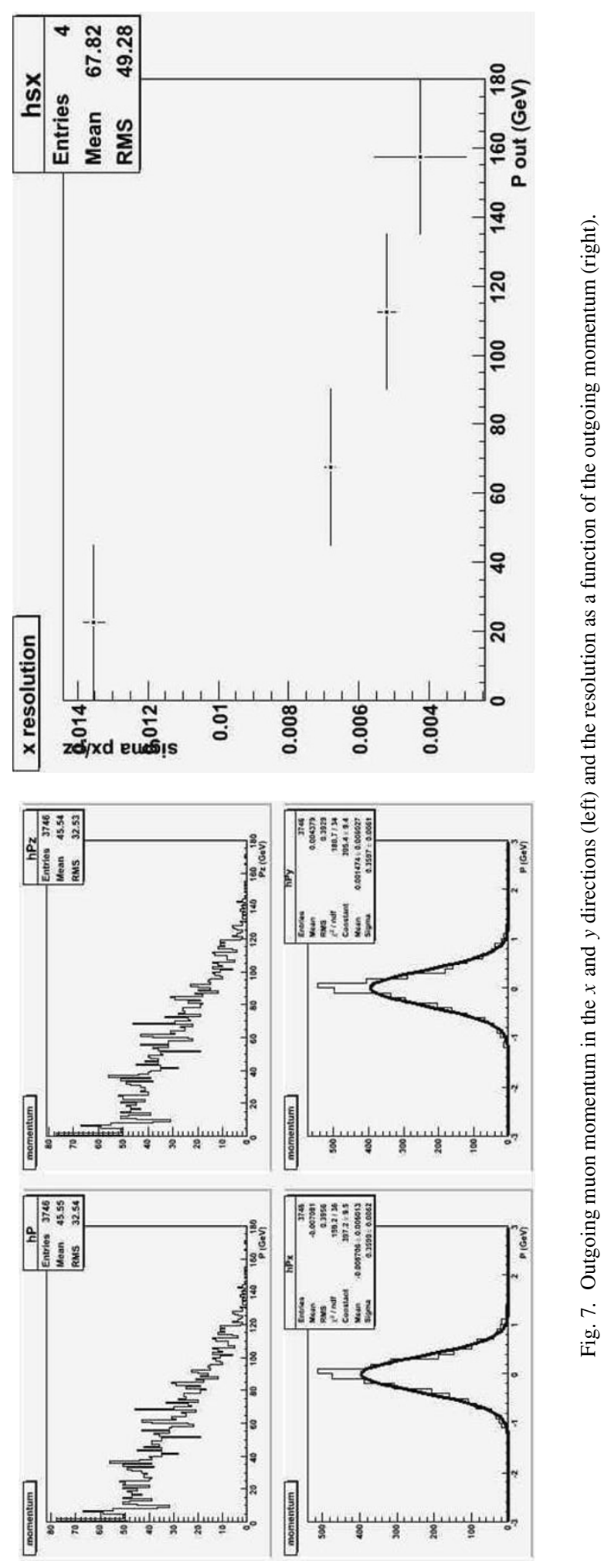


known flux of atmospheric neutrinos, depending on energy and angle. An energy threshold was applied to the muons detected in the fiducial volume.

To simulate the radiography of Earth structures by cosmic ray muons, a beam of muons is tracked while crossing the rock of the considered geological structure. Since the dimensions of the detector are orders of magnitude smaller than the dimensions of the structure that we want to investigate, in first approximation we can consider the detector as pointlike and, by using a Digital Elevation Map, for each direction crossing the detector we can evaluate the amount of rock crossed by the muon. In such a way, as in the case of neutrino Earth radiography, we can define the dimensions of the passive material where GEANT4 performs the tracking. To study the presence of holes or of zones with a different density in the passive material structure, one or more substructure can be added. The output gives information about the outgoing muon energy and direction.

\section{Results and Conclusions}

In Fig. 4 we show number and type of the particles arriving to the NT in the first 2000 events of a GEANT4 run for a primary neutrino of energy $10^{4} \mathrm{GeV}$ : the plot on the left is reported as a contour on the right, where the more large are the circles, the higher is the corresponding number of particles. Moreover, the particles corresponding to some of the particle codes are highlighted. On the other side, Fig. 5 shows the angular distribution of events simulated with our simplified Monte Carlo, and is in nice agreement with the line corresponding to $E_{\text {th }}=1 \mathrm{TeV}$ in figure 1(a) of González-García et al. (2008).

Concerning the muon radiography of geological structures, as first step of our study we performed a very preliminary analysis: we simulated 10000 muons with an energy of $1 \mathrm{TeV}$, in the $z$ direction, and tracked them trough $1 \mathrm{~km}$ of rock with density $2.6 \mathrm{~g} / \mathrm{cm}^{3}$. Only 3746 of these muons were able to cross the whole structure and their angular resolution was $\sim 10 \mathrm{mrad}$, as shown in Fig. 6. In Fig. 7 the momentum of the outgoing muon in the $x$ and $y$ direction and the resolution obtained as a function of the outgoing momentum are shown, respectively. As we can see, the largest contribution to the resolution is due to muons with a momentum below $40 \mathrm{GeV}$.

By summarizing, the results we have presented confirm that neutrino or muon radiography of geological structures is a very promising research field, and with the help of the simulations we are developing one can achieve the final goal of recognizing a non trivial density profile with a good level of statistical confidence.

Acknowledgments. G.M. acknowledges support by the Spanish MICINN (grants SAB2006-0171 and FPA2005-01269). G.M. and O.P. acknowledge support by INFN I.S. Fa51 and PRIN 2006 Fisica Astroparticellare: Neutrini ed Universo Primordiale of Italian MIUR.

\section{References}

Aggouras, G. et al. [NESTOR Collaboration], Recent results from NESTOR, Nucl. Instrum. Meth. A, 567, 452, 2006.

Agostinelli, S. et al., G4-a simulation toolikit, Nuclear Instruments and Methods in Physics Research Section A: Accelerators, Spectrometers, Detectors and Associated Equipment, 506, 205, 2003.
Ahrens, J. et al. [AMANDA Collaboration], Observation of high-energy atmospheric neutrinos with the Antarctic Muon and Neutrino Detector Array, Phys. Rev. D, 66, 012005, 2002.

Ahrens, J. et al. [IceCube Collaboration], Sensitivity of the IceCube detector to astrophysical sources of high energy muon neutrinos, Astropart. Phys., 20, 507, 2004.

Aki, K. and P. G. Richards, Quantitative Seismology: Theory and Methods, vols. 1 and 2, W. H. Freeman, 1980.

Allison, J. et al., Geant4 developments and applications, Nucl. Sci. IEEE Trans., 53, 270, 2006.

Ambrosio, M. et al., Vertical muon intensity measured with MACRO at the Gran Sasso laboratory, Phys. Rev. D, 52, 3793, 1995.

Ambrosio, M., C. Aramo, A. Della Selva, G. Miele, S. Pastor, O. Pisanti, and L. Rosa, Corsika+Herwig Monte Carlo Simulation of neutrino induced atmospheric air showers, AUGER-GAP-2003-013, 2003.

Balkanov, V. A. et al., Registration of atmospheric neutrinos with the BAIKAL neutrino telescope NT-96, Astropart. Phys., 12, 75, 1999.

Corcella, G., I. G. Knowles, G. Marchesini, S. Moretti, K. Odagiri, P. Richardson, M. H. Seymour, and B. R. Webber, HERWIG 6: An Event generator for hadron emission reactions with interfering gluons (including supersymmetric processes), JHEP, 0101, 010, 2001.

Cuoco, A., G. Mangano, G. Miele, S. Pastor, L. Perrone, O. Pisanti, and P. D. Serpico, Ultrahigh energy neutrinos in the Mediterranean: Detecting $\mathrm{v}(\mathrm{tau})$ and $\mathrm{v}(\mathrm{mu})$ with a $\mathrm{km} 3$ telescope, JCAP, 0702, 007, 2007.

Dziewonski, A., Earth structure, global, in The Encyclopedia of Solid Earth Geophysics, edited by James, D. E., Van Nostrand Reinhold, New York, 331, 1971.

ETOPO2, U.S. Department of Commerce, National Oceanic and Atmospheric Administration, National Geophysical Data Center, 2-minute Gridded Global Relief Data, http://www.ngdc.noaa.gov/ mgg/fliers/01mgg04.html, 2001.

Gaisser, T. K. and T. Stanev, Cosmin rays, in Amsler, C. et al. (Particle Data Group), Phys. Lett. B, 667, 1, 2008.

González-García, M. C., F. Halzen, M. Maltoni, and H. K. M. Tanaka, Radiography of Earth's core and mantle with atmospheric neutrinos, Phys. Rev. Lett., 100, 061802, 2008.

Heck, D., G. Schatz, T. Thouw, J. Knapp, and J. N. Capdevielle, CORSIKA, FZKA, 6019, 1998. Forschungszentrum Karlsruhe (web page: http://www-ik3.fzk.de/ heck/corsika).

Jain, P., J. P. Ralston, and G. M. Frichter, Neutrino absorption tomography of the Earth's interior using isotropic ultra-high energy flux, Astropart. Phys., 12, 193, 1999.

Katz, U. F., KM3NeT: Towards a km**3 Mediterranean neutrino telescope, Nucl. Instrum. Meth. A, 567, 457, 2006.

Lay, T. and T. C. Wallace, Modern Global Seismology, Academic Press, 1995.

Miele, G., S. Pastor, and O. Pisanti, The aperture for UHE neutrinos of the Auger fluorescence detector using a Digital Elevation Map, Phys. Lett. $B, \mathbf{6 3 4}, 137,2006$.

Migneco, E. et al. [NEMO Collaboration], Recent achievements of the NEMO project, Nucl. Instrum. Meth. A, 588, 111, 2008.

Nagamine, K., M. Iwasaki, K. Shimomura, and K. Ishida, Method of probing inner structure of geophysical substance with the horizontal cosmic ray muons and possible application to volcanic eruption prediction, Nucl. Instrum. Meth. A, 356, 585, 1995.

Reynoso, M. M. and O. A. Sampayo, On neutrino absorption tomography of the Earth, Astropart. Phys., 21, 315, 2004.

Spurio, M. [ANTARES Collaboration], Status report (2006) of the ANTARES project, 2006.

Tanaka, H. K. M. and I. Yokoyama, Muon radiography and deformation analysis of the lava dome formed by the 1944 eruption of Usu, Hokkaido-Contact between high-energy physics and volcano physics-, Proc. Jpn. Acad. Ser. B, 84, 2008.

Tanaka, H. K. M., K. Nagamine, N. Kawamura, S. N. Nakamura, K. Ishida, and K. Shimomura, Development of a two-fold segmented detection system for near horizontally cosmic-ray muons to probe the internal structure of a volcano, Nucl. Instrum. Meth. A, 507, 657, 2003.

Tanaka, H. K. M., K. Nagamine, S. N. Nakamura, and K. Ishida, Radiographic measurements of the internal structure of Mt. West Iwate with near-horizontal cosmic-ray muons and future developments, $\mathrm{Nucl}$. Instrum. Meth. A, 555, 164, 2005.

Tanaka, H. K. M., T. Nakano, S. Takahashi, J. Yoshida, and K. Niwa, Development of an emulsion imaging system for cosmic-ray muon radiography to explore the internal structure of a volcano, Mt. Asama, Nucl. Instrum. Meth. A, 575, 489, 2007a.

Tanaka, H. K. M., T. Nakano, S. Takahashi, J. Yoshida, M. Takeo, J. 
Oikawa, T. Ohminato, Y. Aoki, E. Koyama, H. Tsuji, and K. Niwa, High resolution imaging in the inhomogeneous crust with cosmic-ray muon radiography: The density structure below the volcanic crater floor of Mt. Asama, Japan, Earth Planet. Sci. Lett., 263, 104, 2007b.

Tanaka, H. K. M., T. Nakano, S. Takahashi, J. Yoshida, H. Ohshima, T. Maekawa, H. Watanabe, and K. Niwa, Imaging the conduit size of the dome with cosmic-ray muons: The structure beneath Showa-Shinzan Lava Dome, Geophys. Res. Lett., 34, 389, 2007c.

Tanaka, H. K. M., T. Nakano, S. Takahashi, J. Yoshida, M. Takeo, J. Oikawa, T. Ohminato, Y. Aoki, E. Koyama, H. Tsuji, H. Ohshima, T.
Maekawa, H. Watanabe, and K. Niwa, Radiographic imaging below a volcanic crater floor with cosmic-ray muons, Am.J. Sci., 308, 843, 2008. Thompson, M. G. and M. R. Whalley, The production spectra of the parents of vertical cosmic ray muons, J. Phys. G: Nucl. Phys., 1, L48-L50, 1975.

E. Borriello, G. De Lellis, G. Mangano, A. Marotta, G. Miele, P. Migliozzi, C. Moura, S. Pastor, O. Pisanti (e-mail: pisanti@na.infn.it), P. Strolin, and A. Zollo 